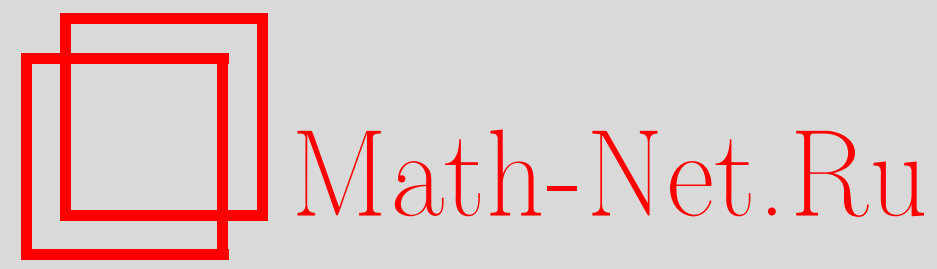

А. В. Лебедев, Предельные теоремы о максимумах пуассоновских последовательностей и их применения в теории массового обслуживания, Теория вероятн. и ее примен., 1999, том 44, выпуск 2, 446-450

DOI: https://doi.org/10.4213/tvp779

Использование Общероссийского математического портала Math-Net.Ru подразумевает, что вы прочитали и согласны с пользовательским соглашением http://www.mathnet.ru/rus/agreement

Параметры загрузки:

IP : 54.198 .55 .26

26 апреля 2023 г., 13:57:36

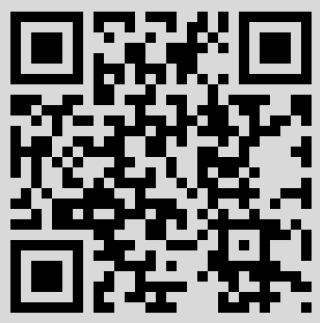


(C) 1999 г.

\title{
ПРЕДЕЛЬНЫЕ ТЕОРЕМЫ О МАКСИМУМАХ ПУАССОНОВСКИХ ПОСЛЕДОВАТЕЛЬНОСТЕЙ И ИХ ПРИМЕНЕНИЯ В ТЕОРИИ МАССОВОГО ОБСЛУЖИВАНИЯ ${ }^{1)}$
}

\begin{abstract}
Рассматривается последовательность независимых пуассоновских случайвых величин $X_{n}$ с параметрами $\lambda_{n}$. Изучаются асимттотические свойства максимумов $Y_{n}=\max _{1 \leqslant i \leqslant n} X_{i}$ в зависимости от свойств последовательности $\left\{\lambda_{n}\right\}$. Полученные результаты применяются к исследованию максимального числа заявок в системе $M|G| \infty$ с параметрами, зависяпими от времени.
\end{abstract}

Ключевые слова и фразы: пуассоновские случайные величины, максимумы, предельные теоремы, сходимость почти наверное ${ }_{4}$ бесконечноливейные системы массового обслуживания.

1. Классы последовательностей. Введем классы неотрицательных числовых последовательностей следующим образом.

О п р е д е л е н и е 1 . Числовая последовательность $\left\{\lambda_{n}\right\}$ принадлежит классу $D$, если существует такое $s>0$, что $\lambda_{n} \leqslant n^{-s}$ при всех достаточно больших $n$.

O п р е д е л е и и е 2. Числовая последовательность $\left\{\lambda_{n}\right\}$ принадлежит классу $L$, если для некоторого $r>0$ и любого $s>0$ верно $\lambda_{n} \leqslant r \ln n$ и $\lambda_{n}>n^{-s}$ при всех достаточно больших $n$.

О п р е д е л е н и е 3 . Числовая последовательность $\left\{\lambda_{n}\right\}$ принадлежит классу $I$, если $\lambda_{n} / \ln n \rightarrow \infty$ при $n \rightarrow \infty$.

2. Свойства максимумов. Как было отмечено еше в [1], для одинаково распределенных независимых пуассоновских случайных величин не существует линейной нормировки, при которой распределения нормированных максимумов сходились бы к некоторому невырожденному предельному закону.

Однако в ряде случаев оказывается возможным получать для нормированных максимумов верхние пределы почти наверное (п.н.).

Пусть $X_{n}$ - независимые пуассоновские случайные величины с параметрамк $\lambda_{n}$, $n \geqslant 1$. Обозначим $Y_{n}=\max _{1 \leqslant i \leqslant n} X_{i}$.

Далее будем пользоваться неравенством

$$
\frac{\lambda^{m}}{m !} \leqslant \sum_{k=m}^{\infty} \frac{\lambda^{k}}{k !} \leqslant\left(1-\frac{\lambda}{m+1}\right)^{-1} \frac{\lambda^{m}}{m !}, \quad \lambda<m+1,
$$

доказательство которого элементарно.

Теорема 1. Eсли $\left\{\lambda_{n}\right\} \in D$, mo $Y=\sup X_{n}-$ собственная случайная величина.

Д ока з ат т льст во.

$$
\begin{aligned}
\mathbf{P}\{Y \leqslant N\} & =\prod_{n=1}^{\infty} \sum_{k=0}^{N} \frac{\lambda_{n}^{k}}{k !} e^{-\lambda_{n}}=\prod_{n=1}^{\infty}\left(1-\sum_{k=N+1}^{\infty} \frac{\lambda_{n}^{k}}{k !} e^{-\lambda_{n}}\right) \\
& \geqslant 1-\sum_{n=1}^{\infty} \sum_{k=N+1}^{\infty} \frac{\lambda_{n}^{k}}{k !} e^{-\lambda_{n}} \geqslant 1-\sum_{n=1}^{\infty} \sum_{k=N+1}^{\infty} \frac{\lambda_{n}^{k}}{k !}
\end{aligned}
$$

* Московский государственный универсктет, механико-математический факультет, кафедра теории вероятностей, Воробьевы горы, 119899 Москва, Россия.

1) Работа выполнена при поддержке Российского фонда фундаментальных исследований, грант 96-01-01092. 
Обозначим $\Lambda=\sup \lambda_{n} n^{s}<\infty$. Тогда при $N>\max (\Lambda, 1 / s)$ имеем:

$$
\begin{aligned}
\sum_{n=1}^{\infty} \sum_{k=N+1}^{\infty} \frac{\lambda_{n}^{k}}{k !} & \leqslant \sum_{n=1}^{\infty}\left(1-\frac{\lambda_{n}}{N+2}\right)^{-1} \frac{\lambda_{n}^{N+1}}{(N+1) !} \\
& \leqslant\left(1-\frac{\Lambda}{N+2}\right)^{-1} \sum_{n=1}^{\infty} n^{-s(N+1)} \frac{\Lambda^{N+1}}{(N+1) !} \rightarrow 0, \quad N \rightarrow \infty .
\end{aligned}
$$

Следовательно, $\mathbf{P}\{Y \leqslant N\} \rightarrow 1$ при $N \rightarrow \infty$, т.е. распределение $Y$ собственное. Теорема доказана.

Перейдем к рассмотрению случаев $\left\{\lambda_{n}\right\} \in L,\left\{\lambda_{n}\right\} \in I$.

Лемма 1. Ec.лu $\limsup \sup _{n \rightarrow \infty} \lambda_{n} / m_{n}<1$, mo pяdb $\sum_{n=1}^{\infty} \mathbf{P}\left\{X_{n} \geqslant m_{n}\right\}$ u $\sum_{n=1}^{\infty} \mathbf{P}\left\{X_{n}=m_{n}\right\}$ сходятся или растодятся одновременно.

Д о к а з ат е л в с т в о. Утверждение леммы непосредственно следует из неравенства (1).

Обозначим $\varphi(x)=x(\ln x-1)+1, x \geqslant 1$. Функция $\varphi$ монотонна и $\varphi:[1,+\infty) \rightarrow$ $[0,+\infty)$. Следовательно, существует обратная функция $\varphi^{-1}:[0,+\infty) \rightarrow[1,+\infty)$.

Обозначим $\tau(\theta, x)=\varphi\left(\theta \varphi^{-1}(x)\right) / x$, где $\theta>0, x>0$ и $\varphi^{-1}(x) \geqslant 1 / \theta$. Очевидно, $\tau(1, x) \equiv 1$ и $\tau$ монотонна по $\theta$.

Лемма 2. При иобом $x_{0}>0$ для всех $x \geqslant x_{0}$ выполняются неравенства:

1) $\tau(\theta, x) \geqslant \theta$ прu $\theta>1$,

2) $\tau(\theta, x) \leqslant 1-c\left(\theta, x_{0}\right)(1-\theta)$ npu $\theta_{0}<\theta<1$, где $c\left(\theta, x_{0}\right)=1-\max \left\{0, \ln \theta / \ln \theta_{0}\right\}>0$, $\theta_{0}=1 / \varphi^{-1}\left(x_{0}\right)$.

Д о к а за т е л с с т в о. При $x \geqslant x_{0}, \theta>\theta_{0}$ выполнено $\varphi^{-1}(x) \geqslant 1 / \theta$, так что $\tau(\theta, x)$ определено. Будем обозначать $y=\varphi^{-1}(x) \geqslant 1 / \theta_{0}>1$. Рассмотрим производную

$$
\frac{\partial \tau}{\partial \theta}(\theta, x)=\frac{\varphi^{-1}(x)}{x} \ln \left(\theta \varphi^{-1}(x)\right)=\frac{y}{\varphi(y)} \ln \theta y \geqslant \frac{\ln \theta y}{\ln y} \geqslant c\left(\theta, x_{0}\right) .
$$

При $\theta>1$ получаем $c\left(\theta, x_{0}\right) \equiv 1$. Воспользуемся теоремой Лагранжа о конечных приращениях: $\tau(\theta, x)=1+(\partial \tau / \partial \theta)(\xi, x)(\theta-1)$, где $\xi$ лежит на интервале между $\theta$ и 1 . При $\theta>1$ получаем отскда $\tau(\theta, x) \geqslant \theta$, а при $\theta_{0}<\theta<1$ имеем $c\left(\xi, x_{0}\right) \geqslant c\left(\theta, x_{0}\right)$ и $\tau(\theta, x) \leqslant 1-c\left(\theta, x_{0}\right)(1-\theta)$.

Обозначим $\varepsilon_{n}=(\ln n) / \lambda_{n}, \lambda_{n}>0$ и $u_{n}=\lambda_{n} \varphi^{-1}\left(\varepsilon_{n}\right)$.

Лемма 3. Ecлu $\left\{\lambda_{n}\right\} \in L$, то $u_{n} \rightarrow \infty, n \rightarrow \infty$.

Д о х а з а т е л в с т в о. Для функцик $\varphi^{-1}$ имеет место оценка:

$$
\varphi^{-1}(x) \geqslant \frac{x+1}{\ln (x+1)+1}, \quad \forall x \geqslant 0,
$$

что доказывается непосредственной подстановкой правой части в $\varphi$.

По определению класса $L$ для любого $s>0$ при всех достаточно больших $n$ верно $\lambda_{n}>n^{-s}$. Следовательно,

$$
\liminf _{n \rightarrow \infty} u_{n} \geqslant \lim _{n \rightarrow \infty} \frac{\ln n+n^{-s}}{\ln \left(n^{s} \ln n+1\right)+1}=\frac{1}{s}, \quad \forall s>0,
$$

что и дохазывает лемму.

Заметим, что имеет место асимптотика $\varphi^{-1}(x) \sim x / \ln x, x \rightarrow \infty$. При $\lambda_{n}=o(\ln n)$ получаем

$$
u_{n} \sim \frac{\ln n}{\ln \varepsilon_{n}}=\frac{\ln n}{\ln \ln n-\ln \lambda_{n}}, \quad n \rightarrow \infty .
$$

Лемма 4. Eсли $\left\{\lambda_{n}\right\} \in L$, то сущестөует такое $\theta_{0}$, что $\partial_{s} m_{n} \sim \theta u_{n}, n \rightarrow \infty$, pяd $\sum_{n=1}^{\infty} P\left\{X_{n}=m_{n}\right\}$ pacxodumcs npu $\theta_{0}<\theta<1$ u cxodumcr npu $\theta>1$.

Д о ка зате льст в о. При всех достаточно больших $n$ имеем $\varepsilon_{n} \geqslant 1 / r>0$. Положим $\theta_{0}=1 / \varphi^{-1}(1 / r)$. Воспользовавшись формулой Стирлинга, получим

$$
\begin{aligned}
\ln \mathbf{P}\left\{X_{n}=m_{n}\right\} & =-\lambda_{n}+m_{n} \ln \lambda_{n}-m_{n} \ln m_{n}+m_{n}-\frac{1}{2} \ln m_{n}+O(1) \\
& \sim-\tau\left(\theta, \varepsilon_{n}\right) \ln n, \quad n \rightarrow \infty .
\end{aligned}
$$


Применяя лемму 2, интегральный признак Коши и признак сравнения рядов, получаем утверждение леммы.

Будем пользоваться утверждением теоремы 4.4.1 [2, с. 201] в следующей формулировке: если $X_{n}$ - независимые случайные величины, $Y_{n}=\max _{1 \leqslant i \leqslant n} X_{i}, u_{n}-$ неубывающая числовая последовательность, $u_{n} \rightarrow \infty$ при $n \rightarrow \infty$ и $S=\{\theta>$ $0: \sum_{n=1}^{\infty} \mathbf{P}\left\{X_{n}>\theta u_{n}\right\}$ расходится $\}$, то

$$
\limsup _{n \rightarrow \infty} \frac{Y_{n}}{u_{n}} \stackrel{\text { n.H. }}{=} \begin{cases}\sup S, & S \neq \varnothing, \\ 0, & S=\varnothing .\end{cases}
$$

Можно показать, что условие неубывания $u_{n}$ здесь можно заменить на условие асимптотической эквивалентности неубывающей последовательности. Будем обозначать класс последовательностей, удовлетворяющих такому условию, через $A$.

Теорема 2. Ec.лu $\left\{\lambda_{n}\right\} \in L,\left\{u_{n}\right\} \in A, \operatorname{mo} \lim \sup _{n \rightarrow \infty} Y_{n} / u_{n}=1$ n.н.

Д о к а з а т е л в с т в о. Пусть $m_{n}=\left[\theta u_{n}\right]+1, \theta>\theta_{0}$. По лемме 3 имеем $u_{n} \rightarrow \infty$. По лемме 4 ряд $\sum_{n=1}^{\infty} \mathbf{P}\left\{X_{n}=m_{n}\right\}$ расходится при $\theta_{0}<\theta<1$ и сходится при $\theta>1$, а по лемме 1 ряд $\sum_{n=1}^{\infty} \mathbf{P}\left\{X_{n} \geqslant m_{n}\right\}=\sum_{n=1}^{\infty} \mathbf{P}\left\{X_{n}>\theta u_{n}\right\}$ сходится или расходится одновременно с ним. Отсюда следует утверждение теоремы.

Теорема 3. Ecли $\left\{\lambda_{n}\right\} \in I \cap A$, mo $\lim \sup _{n \rightarrow \infty} Y_{n} / \lambda_{n}=1$ п.к.

Д о к а з а т е л ь с т в о. Пусть $m_{n}=\left[\theta \lambda_{n}\right]+1$, где $\theta>1$, тогда $\ln \mathbf{P}\left\{X_{n}=\right.$ $\left.m_{n}+1\right\} \sim-\lambda_{n} \varphi(\theta), n \rightarrow \infty$. При достаточно больших $n$ имеем $\lambda_{n}>(2 / \varphi(\theta)) \ln n$, так что ряд $\sum_{n=1}^{\infty} \mathbf{P}\left\{X_{n}=m_{n}\right\}$ сходится, а по лемме 1 сходится и ряд $\sum_{n=1}^{\infty} \mathbf{P}\left\{X_{n} \geqslant m_{n}\right\}$. Значит, $\limsup \operatorname{su}_{n \rightarrow \infty} Y_{n} / \lambda_{n} \leqslant 1$. С другой стороны, при $\lambda_{n} \rightarrow \infty$ можно воспользоваться асимптотической нормальностью $X_{n}$ и получить $\mathbf{P}\left\{X_{n}>\lambda_{n}\right\} \rightarrow \frac{1}{2}$, откуда следует, что ряд $\sum_{n=1}^{\infty} \mathbf{P}\left\{X_{n}>\lambda_{n}\right\}$ расходится и $\lim \sup _{n \rightarrow \infty} Y_{n} / \lambda_{n} \geqslant 1$. Таким образом, утверждение теоремы доказано.

3. Примеры. В качестве приложения полученных результатов рассмотрим следующие примеры.

3.1. Пусть $\lambda_{n}=\lambda$, тогда по (2) и теореме 2

$$
\limsup _{n \rightarrow \infty} Y_{n} \frac{\ln \ln n}{\ln n}=1 \quad \text { п.н. }
$$

Этот известный ранее результат упомянут, например, в [2].

3.2. Пусть $\lambda_{n} \sim r \ln n, n \rightarrow \infty$, тогда по теореме 2

$$
\limsup _{n \rightarrow \infty} \frac{Y_{n}}{r \varphi^{-1}(1 / r) \ln n}=1 \quad \text { п.н. }
$$

3.3. Пусть $\lambda_{n} \sim c n, n \rightarrow \infty$, тогда по теореме 3

$$
\limsup _{n \rightarrow \infty} Y_{n} / \lambda_{n}=1 \text { п.н. }
$$

4. Применения в теории массового обслуживания. Рассмотрим систему с нестационарным пуассоновским входным потоком интенсивности $\lambda(t)$ и бесконечным числом приборов. Если заявка приходит в момент $t$, то время ее обслуживания имеет функцию распределения $B_{t}(x)$. Обслуживание заявок происходит независимо, в начальный момент система свободна.

Подобные системы рассматриваются, например, в [3], [4].

Нас будет интересовать асимптотическое поведение максимума $M(t)$ числа заявок в системе на $[0, t]$ при $t \rightarrow \infty$.

Обозначим через $\prec$ отношение «стохастически меньше», т.е. $\xi \prec \eta \Leftrightarrow \mathbf{P}\{\xi>$ $x\} \leqslant \mathbf{P}\{\eta>x\}$.

Лемма 5. Пусть $\left\{v_{n}\right\}-$ случайная последовательность, $\Upsilon_{n}=\max _{1 \leqslant i \leqslant n} v_{i} u$

1) $v_{n}^{\prime} \leqslant v_{n}$, где $v_{n}^{\prime}$ кезависчмы, $\Upsilon_{n}^{\prime}=\max _{1 \leqslant i \leqslant n} v_{i}^{\prime}$,

2) $v_{n} \prec v_{n}^{\prime \prime}$, аде $v_{n}^{\prime \prime}$ независимы, $\Upsilon_{n}^{\prime \prime}=\max _{1 \leqslant i \leqslant n} v_{i}^{\prime \prime} ;$ погда для любой числовой последовательности $\left\{u_{n}\right\} \in A, u_{n} \rightarrow \infty, n \rightarrow \infty$, выполнено керавенство

$$
\limsup _{n \rightarrow \infty} \frac{\Upsilon_{n}^{\prime}}{u_{n}} \leqslant \limsup _{n \rightarrow \infty} \frac{\Upsilon_{n}}{u_{n}} \leqslant \limsup _{n \rightarrow \infty} \frac{\Upsilon_{n}^{\prime \prime}}{u_{n}} \quad \text { n.H. }
$$


Д о к а з а т е л ь с т в о. Оценка снизу для $\lim \sup _{n \rightarrow \infty} \Upsilon_{n} / u_{n}$, очевкдно, следует из условия 1). Если $l=\lim \sup _{n \rightarrow \infty} \Upsilon_{n}^{\prime \prime} / u_{n}<\infty$, то из условия 2) для любого $\varepsilon>0$ получаем: $\sum_{n=1}^{\infty} \mathbf{P}\left\{v_{n}>(l+\varepsilon) u_{n}\right\} \leqslant \sum_{n=1}^{\infty} \mathbf{P}\left\{v_{n}^{\prime \prime}>(l+\varepsilon) u_{n}\right\}<\infty$, откуда по теореме $4.2 .1\left[2\right.$, с. 189] следует $\lim \sup _{n \rightarrow \infty} \Upsilon_{n} / u_{n} \leqslant l+\varepsilon$, что и доказывает лемму.

Пусть $0=t_{0}<t_{1}<\cdots<t_{n}<\cdots-$ числовая последовательность, стремящаяся к бесконечности. Обозначим через $\mu_{n}$ максимальное число заявок на отрезке $J_{n}=$ $\left[t_{n-1}, t_{n}\right]$, через $\mu_{n}^{\prime}$ - число заявок, пришедших в течение $J_{n}$ и оставшихся $\mathbf{k}$ его концу, и через $\mu_{n}^{S}-$ сумму числа заявок, находившихся в системе в момент $t_{n-1}$, и числа заявок, пришедших в течение $J_{n}$. Очевидно, $\mu_{n}^{\prime} \leqslant \mu_{n} \leqslant \mu_{n}^{S}$, причем $\mu_{n}^{\prime}$ независимы.

Построим последовательность независимых случайных величин $\mu_{n}^{\prime \prime} \stackrel{d}{=} \mu_{n}^{S}$. Тогда $\mu_{n}^{\prime} \leqslant \mu_{n} \prec \mu_{n}^{\prime \prime}$, т.е. выполняются условия леммы 5. Заметим, что $\mu_{n}^{\prime}$ и $\mu_{n}^{\prime \prime}-$ пуассоновские случайные величины с параметрами

$$
\begin{aligned}
& \lambda_{n}^{\prime}=\int_{t_{n-1}}^{t_{n}} \lambda(u) \bar{B}_{u}\left(t_{n}-u\right) d u, \\
& \lambda_{n}^{\prime \prime}=\int_{0}^{t_{n-1}} \lambda(u) \bar{B}_{u}\left(t_{n-1}-u\right) d u+\int_{t_{n-1}}^{t_{n}} \lambda(u) d u
\end{aligned}
$$

соответственно. Изучая асимптотику случайных последовательностей $M_{n}^{\prime}=$ $\max _{1 \leqslant i \leqslant n} \mu_{i}^{\prime}$ и $M_{n}^{\prime \prime}=\max _{1 \leqslant i \leqslant n} \mu_{i}^{\prime \prime}$, можно получить утверждения об асимптотике $M\left(t_{n}\right)=\max _{1 \leqslant i \leqslant n} \mu_{i}$.

Следующая теорема иллюстрирует применение описанного выше метода в случае, когда распределение времени обслуживания не зависит от момента поступления заявки и имеет конечное среднее $\beta$, а интенсивность входного потока растет быстрее логарифма, но медленнее экспоненты.

Теорема 4. Пусть $\lambda(t)$ не убывает при всех достаточно больщих $t \quad u$ $\lambda(t) / \ln t \rightarrow \infty, \lambda(t+p) / \lambda(t) \rightarrow 1, \forall p$ npu $t \rightarrow \infty$, mozda

$$
\limsup _{t \rightarrow \infty} \frac{M(t)}{\lambda(t)}=\beta \quad \text { n.н. }
$$

Дока за те ль с т в о. Положим $t_{n}=n h, h>0, n \geqslant 0$. Из (3) и условия теоремы получаем для всех достаточно больших $n: \lambda_{n}^{\prime} \geqslant \lambda\left(t_{n-1}\right) \int_{0}^{h} \bar{B}(u) d u, \lambda_{n}^{\prime \prime} \leqslant$ $\lambda\left(t_{n}\right)(\beta+h)$. Применяя лемму 5 и теорему 3 , получаем:

$$
\int_{0}^{h} \bar{B}(u) d u \leqslant \limsup _{n \rightarrow \infty} \frac{M_{n}^{\prime}}{\lambda\left(t_{n}\right)} \leqslant \limsup _{n \rightarrow \infty} \frac{M\left(t_{n}\right)}{\lambda\left(t_{n}\right)} \leqslant \limsup _{n \rightarrow \infty} \frac{M_{n}^{\prime \prime}}{\lambda\left(t_{n}\right)} \leqslant \beta+h .
$$

При любом $h>0$ имеем

$$
\limsup _{n \rightarrow \infty} \frac{M\left(t_{n}\right)}{\lambda\left(t_{n}\right)}=\limsup _{t \rightarrow \infty} \frac{M(t)}{\lambda(t)} .
$$

Переходя в левой части неравенства (5) к пределу при $h \rightarrow \infty$, а в правой части при $h \rightarrow 0$, получаем утверждение теоремы.

Заметим, что (4) можно обратить следующим образом: пусть $T_{L}$ - момент первого достижения числом заявок уровня $L$, тогда $\liminf \lim _{L \rightarrow \infty} \lambda\left(T_{L}\right) / L=1 / \beta$ п.н.

Автор благодарен Е.В. Булинской и А. В. Печинкину за полезные замечания при обсуждении.

\section{СПИСОК ЛИТЕРАТУРЫ}

1. Gnedenko $B$. V. Sur la distribution limite du terme maximum d'une série aléatoire. Ann. Math., 1943, v. 44, № 3, p. 423-453.

2. Галамбои Я. Асимптотическая теория экстремальных порядковых статистик. М.: Наука, 1984. $368 \mathrm{c}$. 
3. Freyer $B$. Ein Bedienungssystem $(M|G| \infty)$ mit zeitabhängiger Eingangsintensität und Bedienungszeitverteilung. - Math. Operationsforsch. Statistik, 1974, B. 5, H. 9, S. 701-708.

4. Аядронов $A . M$. Анализ нестащионарной бесконечнолинейной системы массового обслуживания как модели сети случайного множественного доступа. - Автомат. и вычисл. техника, 1994, № 1 , с. 28-33.

Поступила в редакцию

4.XI.1996

Переработанный вариант

21.X.1998

(C) $1999 \mathrm{r}$.
HACЫРОВ $\Phi$. C.*
О ПРЕДСТАВЛЕНИИ НЕКОТОРЫХ КЛАССОВ СТОХАСТИЧЕСКИХ ИНТЕГРАЛОВ ИТО В ВКДЕ ПІОТРАЕКТОРНЫХ ИНТЕГРАЛОВ ЛЕБЕГА ${ }^{1)}$

\begin{abstract}
Получено представление интеграла Ито в виде интеграла Лебега и обобщение формулы Ито. Доказано, что мовотонная перестановка траекторий виверовското процесса абсолютно непрерывна.
\end{abstract}

Ключевые слова и фразы: интеграл Ито, локаљные времена, формула Ито, броуновское движение.

1. Основные результаты. Пусть на вероятностном пространстве $(\Omega, \mathscr{F}, \mathbf{P})$ задан стандартный винеровский процесс $X(s), s \in[0,1]$. Положим $M(t)=$ $\max \{X(s): s \in[0, t]\}, m(t)=\min \{X(s): s \in[0, t]\}, 0 \leqslant t \leqslant 1$. Пусть $\alpha(t, u), t \in[0,1]$, $u \in \mathbf{R}=(-\infty,+\infty),-$ лохальное время процесса броуновского движения, непрерывное с вероятностью 1 по переменным $(t, u)$. Из определения локального времени следует (см. [2]), что для любой ограниченной (или знакопостоянной) борелевской функции $f(\cdot, \cdot)$ справедливо равенство

$$
\int_{0}^{t} f(s, X(s)) d s=\int_{m(t)}^{M(t)} \int_{0}^{t} f(s, u) \alpha(d s, u) d u .
$$

Обозначим через $\not 5 \sigma$-алгебру предсказуемых множеств, а $\sigma$-алгебру борелевских множеств пространств $E=\mathbf{R}$ или $E=\mathbf{R} \times[0,+\infty)$ будем обозначать через $\mathscr{B}(E)$.

В работе для определенного класса подынтегральных функций найдено представление стохастического интеграла Ито в виде интеграла Лебега, получены обобштения классической формулы Ито и доказана абсолютная непрерывность монотонных перестановох реализаций винеровското процесса.

Пусть $\varphi(s, \omega, u)=\varphi(s, u)$ - случайная функция, неотрицательная, ограниченная на множестве $[0,1] \times \Omega \times \mathbf{R}$, измеримая относительно $\boldsymbol{\sigma}$-алгебры $\mathscr{P} \times \mathscr{B}(\mathbf{R})$, положим $\delta(t, u)=\int_{0}^{t} \varphi(s, u) \alpha(d s, u)$. Обозначим при каждом $u \in \mathbf{R}$ и $x \in[0,+\infty)$ через $t^{*}(x, u)$ момент первого попадания фунхиии $\delta(t, u)$ на уровень $x: t^{*}(x, u)=$ $\inf \{s \in[0,1]: \delta(s, u) \geqslant x\}$, если $\delta(t, u) \geqslant x$, и $t^{*}(x, u)=\infty$ в противном случае, $t^{*}(\tau)=t^{*}(\delta(\tau, X(\tau)), X(\tau))$. Рассмотрим ограниченную, измеримую по всем переменным и суммируемую по переменной $v$ функцию $b(v, u, x),(v, u, x) \in \mathbf{R} \times \mathbf{R} \times[0,+\infty)$,

* Уфимский государственный авиационный технический университет, кафедра математики, ул. К. Маркса, 12, 450000 Уфа-Дентр; e-mail: farsag@math.ugatu.ac.ru

1) Работа выполнена при частичной финансовой поддержке Российского фонда фундаментальных исследований, грант № 96-01-00096. 